\section{DO- Osteopathie}

Offizielles Organ folgender Verbände

Verband der Osteopathen Deutschland e.V. (VOD)

Deutsche Akademie für Osteopathische Medizin e.V. (DAOM)

D Association Luxembourgeoise des Ostéopathes (A.L.D.O.)

ISSN 1610-5044

2/2004, 2. Jahrgang

Herausgeber :

Prof. Dr. rer. nat. med. habil. Rainer Breul D.O.h.c.

Marina Fuhrmann D.O. M.R.O

Prof. Dr. med. habil. Karl-Ludwig Resch

Dr. med. Roger Seider D.O.

Verlag

Hippokrates Verlag in

MVS Medizinverlage Stuttgart GmbH \& Co.KG

Oswald-Hesse-Str. 50, 70469 Stuttgart

Redaktion (v.i.S.d.P.)

Marina Horbatsch

Fon/Fax: (07 11) 8931-715/-705

E-Mail: marina.horbatsch@medizinverlage.de

Christoph Newiger in team $4 \mathrm{U}$ Medienbüro

Fraunhoferstr. 42, 80469 München

Fon/Fax: (089) 209001-67/-68

E-Mail: redaktion.do@medizinverlage.de

Marketing:

Marion Krubasik

Fon/Fax: (07 11) 8931-735/-706

E-Mail: marion.krubasik@medizinverlage.de

Anzeigen:

Martina Urban, MVS Mediaservice

Fon/Fax: (07 11) 8931-734/-706

E-Mail: martina.urban@medizinverlage.de

Abonnementservice

Fon/Fax: (07 11) 8931-321/-422

E-Mail: aboservice@thieme.de

Probeabonnement

Fon/Fax: (07 11) 8931-906/-901

E-Mail: kundenservice@thieme.de

Produktion

Satz \& Repro: Fotosatz Sauter, Donzdor

Druck \& Bindung: Rondo Druck, Ebersbach

Autorenhinweise

Auf Anfrage bei der Redaktion.

Urheberrecht:

Mit der Annahme eines Manuskriptes erwirbt der Verlag fü die Dauer der gesetzlichen Schutzfrist ( $\S 64$ UrhRG) die ausschließliche Befugnis zur Wahrnehmung der Verwertungsrechte im Sinne der $\S \S 15$ ff. des Urheberrechtsgesetzes. Die Zeitschrift und alle in ihr enthaltenen einzelnen Beiträge und Abbildungen sind für die Dauer des Urheberrechts geschützt. Jede Verwertung ist ohne Zustimmung des Verlages außerhalb der engen Grenzen des UrhRG unzulässig und strafbar. Dies gilt insbesondere für Vervielfältigungen, Übersetzungen, Microverfilmungen und die Einspeicherung und Verarbeitung in elektronischen Systemen. Für den persönlichen Gebrauch dürfen von Beiträgen oder Teilen von diesen einzelne Kopien hergestellt werden. Die Rechte an den Abbildungen liegen

- wenn nicht anders gekennzeichnet - beim Verlag.

Erscheinungsweise:

Vierteljährlich

Bezugspreise

Abonnement- Versand-

preis koste

Inland $€ 64,90 \quad € 6,50 \quad € 71,40$

Europa $€ 64,90 \quad € 11,20 \quad € 76,10$

Restl. Welt $€ 64,90 \quad € 19,90 \quad € 84,80$

Aus-, Fort- und Weiterbildung, Studenten und AIP:

Inland $€ 39,90 \quad € 6,50 \quad € 46,40$

Europa $€ 39,90 \quad € 11,20 \quad € 51,10$

Restl. Welt $€ 39,90 \quad € 19,90 \quad € 59,80$

Einzelheft $€ 18,00$ zzgl. Versandkosten ab Verlagsort. Alle Preise sind unverbindlich empfohlene Preise. Alle Preise und Versandspesen enthalten 7\% MwSt.

\section{Praxis für Fango und Osteopathie}

L andauf landab wird fleißig umbenannt. Die „Praxis für Physiotherapie und Osteopathie“ - Physiotherapie und Osteopathie eventuell auch in umgekehrter Reihenfolge - entwickelt sich zum Renner. PraxisinhaberInnen mit und ohne DO bieten die Osteopathie oder einzelne osteopathische Verfahren an neben Schlingentisch, Bobath, Shiatsu, Bindegewebsmassagen und Naturmoorpackungen. Die Qualifikation der Anbieter ist dabei häufig (absichtlich?!) nicht erkennbar. Bei unvoreingenommenen Betrachtern/Patienten entsteht darüber hinaus der Eindruck, dass es sich bei der Osteopathie um eine unter vielen Spezialisierungen der Physiotherapie handelt. Natürlich klären die Inhaber der Praxen in obligatorischen Flyern darüber auf, dass es sich bei dieser ganzheitlichen Methode um etwas ganz besonderes handelt - aber irgendwie scheint sich der erste Eindruck oft recht hartnäckig zu halten: Osteopathie muss eine besondere Physiotherapie sein.

Das Problem ist klar: nach häufig jahrelanger Ausbildung sowie Examina unter Schweiß und Tränen hat man sich beruflich intensiv weiterentwickelt und will einerseits signalisieren, dass man etwas Herausragendes zu bieten hat. Andererseits kann man aber die Praxis, die man mühsam aufgebaut hat, nicht einfach verkaufen. Man fühlt sich verantwortlich gegenüber Mitarbeitern und Angestellten, zahlt Kredite ab oder die Osteopathie alleine ernährt noch nicht die Familie. Die räumliche Trennung von osteopathischer und physiotherapeutischer Praxis gestaltet sich in der Regel kostenintensiv.

Die Problematik dieser „Doppelpraxen“ muss natürlich auf mehreren Ebenen gelöst werden. Eine gesetzliche Regelung der Ausbildung und ihrer Abschlüsse ist dringend erforderlich. Wer darf Osteopathie ausüben und wer die Indikation dazu stellen? Muss in Zukunft wirklich erst die Heilpraktikerprüfung abgelegt werden um als Osteopathin oder Osteopath Heilkunde ausüben zu dürfen? Auch bezüglich der Vergütung, bei der momentan noch die phantastischsten Spannen zu verzeichnen sind, sind Regelungen erforderlich, um Planungssicherheit für Anbieter und Patienten zu schaffen.

Leider besteht jedoch die Gefahr, dass die „Praxis für Osteopathie und ..." sich als Dauereinrichtung etabliert und damit die Konturen verschwimmen. Es sollte klar ersichtlich sein, dass die Osteopathie nicht ein Teil der Physiotherapie ist (und somit als diese auch nicht verordnungsfähig), sondern eine eigenständige Ausübung von Heilkunde.

Damit kein einseitiger Eindruck entsteht, muss natürlich erwähnt werden, dass auch Heilpraktiker den rechtsfreien Raum ausnutzen und nach wenigen Einführungskursen "Osteopathie“ anbieten und abrechnen.

Wir leben in einer Zeit des Überganges und das ist ja auch spannend. Vielleicht werden wir eines Tages, wenn alles genau reguliert ist, uns gerade von diesen Vorgaben erdrückt fühlen. Die Regulierungswut der deutschen Bürokratie ist ja sprichwörtlich, und die endgültigen Verordnungen werden sicher nicht dem entsprechen, was man sich aus der Sicht der Osteopathen idealerweise vorstellt. Aber es ist auch wichtig, in sich so klar wie möglich zu bleiben.

Die Herausgeber 